\title{
A unified approach to Hamiltonian systems, Poisson systems, gradient systems, and systems with Lyapunov functions and/or first integrals
}

\author{
Robert I. McLachlan \\ Mathematics Department, Massey University, Palmerston North, New Zealand \\ G.R.W. Quispel \\ Department of Mathematics, LaTrobe University, Bundoora, Melbourne 3083, Australia \\ Nicolas Robidoux \\ Mathematics Department, Massey University, Palmerston North, New Zealand
}

\begin{abstract}
Systems with a first integral (i.e., constant of motion) or a Lyapunov function can be written as "linear-gradient systems" $\dot{x}=L(x) \nabla V(x)$ for an appropriate matrix function $L$, with a generalization to several integrals or Lyapunov functions. The discrete-time analogue, $\Delta x / \Delta t=L \bar{\nabla} V$ where $\bar{\nabla}$ is a "discrete gradient," preserves $V$ as an integral or Lyapunov function, respectively.

02.60.Lj,03.20.+i,11.30.-j
\end{abstract}

Typeset using REVTEX 


\section{INTRODUCTION}

Integrals and Lyapunov functions-quantities that are conserved or dissipated, respectively - are fundamental in dynamics. They severely constrain the system's evolution and can be used to establish stability. There is no universal method to find such quantities, but if they are known (e.g., on physical grounds) we show that the system can be presented in a universal form which makes the conservation/dissipation property manifest. Although elementary, this result is very general and will find many applications: here we use it to preserve the conservation/dissipation property under time discretization.

We start with the definition and an example of each of the classes of systems covered in this Letter.

(i) Hamiltonian systems.

Hamiltonian systems are ubiquitous in physics [1]. They have the form $\dot{x}=J \nabla V(x)$, $x \in \mathbb{R}^{2 n}$, where $V(x)$ denotes the Hamiltonian function, and $J:=\left(\begin{array}{cc}0 & \text { Id } \\ -\operatorname{Id} & 0\end{array}\right)$, where Id denotes the identity matrix in $\mathbb{R}^{n}$.

Example 1. A simple Hamiltonian system is the pendulum [1] $\dot{x}_{1}=x_{2}, \dot{x}_{2}=-\sin \left(x_{1}\right)$; here $n=1$ and $V\left(x_{1}, x_{2}\right)=\frac{1}{2} x_{2}^{2}-\cos \left(x_{1}\right)$.

(ii) Poisson systems.

Poisson systems also occur very frequently in phys-ics ( [1], App. 14). They have the form $\dot{x}=\Omega(x) \nabla V(x), x \in \mathbb{R}^{n}$, where $V(x)$ again denotes the Hamiltonian function and the Poisson structure $\Omega(x)$ is an antisymmetric matrix $\left(\Omega^{t}(x)=-\Omega(x)\right)$, satisfying the Jacobi identity $\Omega_{j k} \partial_{k} \Omega_{\ell m}+\Omega_{\ell k} \partial_{k} \Omega_{m j}+\Omega_{m k} \partial_{k} \Omega_{j \ell}=0$.

Example 2. The equations of motion of a free rigid body with moments of inertia $I_{1}, I_{2}$, and $I_{3}$ form a Poisson system with angular momentum $x \in \mathbb{R}^{3}$ and Poisson structure 


$$
\Omega(x)=\left(\begin{array}{rrr}
0 & x_{3} & -x_{2} \\
-x_{3} & 0 & x_{1} \\
x_{2} & -x_{1} & 0
\end{array}\right)
$$

and Hamiltonian $V(x)=\frac{1}{2} \sum_{i=1}^{3} x_{i}^{2} / I_{i}$. (Actually this is an example of a so-called Lie-Poisson structure, in which $\Omega(x)$ is a linear function.)

(iii) Systems with a first integral.

The ordinary differential equation (ODE) $\dot{x}=f(x), x \in \mathbb{R}^{n}$, is said to have the first integral $V$ if $d V(x) / d t=0$.

Example 3. A Lotka-Volterra system [5]. The ODE

$$
\dot{x}_{1}=e^{x_{3}}, \dot{x}_{2}=e^{x_{1}}+e^{x_{3}}, \dot{x}_{3}=B e^{x_{1}}+e^{x_{2}},
$$

where $B$ is a parameter, possesses the integral

$$
V\left(x_{1}, x_{2}, x_{3}\right)=e^{x_{2}-x_{1}}+B\left(x_{2}-x_{1}\right)-x_{3} .
$$

(iv) Gradient systems.

Gradient systems arise, e.g., in dynamical systems theory [9]. They are described by $\dot{x}=-\nabla V(x), x \in \mathbb{R}^{n}$.

Example 4. The system $\dot{x}_{1}=-2 x_{1}\left(x_{1}-1\right)\left(2 x_{1}-1\right), \dot{x}_{2}=-2 x_{2}$ is a gradient system [9], with $n=2$ and $V\left(x_{1}, x_{2}\right)=x_{1}^{2}\left(x_{1}-1\right)^{2}+x_{2}^{2}$.

(v) Systems with a Lyapunov function.

The ODE $\dot{x}=f(x), x \in \mathbb{R}^{n}$, is said to possess the Lyapunov function $V$ if $d V(x) / d t \leq$ 0. These functions were introduced by Lyapunov [13] and are a crucial ingredient of his direct or second method in the study of dynamical stability [6, 18.

Example 5. 19. 


$$
\dot{x}_{1}=-x_{2}-x_{1}^{3}, \quad \dot{x}_{2}=x_{1}-x_{2}^{3}
$$

has the Lyapunov function

$$
V\left(x_{1}, x_{2}\right)=x_{1}^{2}+x_{2}^{2}
$$

What do the above five classes of dynamical systems have in common? A preliminary answer would be that they all possess a function $V(x)$ such that $d V(x) / d t \leq 0$. That is, classes (i), (ii) and (iii) each possess a function $V(x)$ such that $d V(x) / d t \equiv 0$, and classes (iv) and (v) each possess a function $V(x)$ s.t. $d V(x) / d t \leq 0$.

In Section 2 we announce the result that classes of systems (i) to (v) have even more in common: under some mild technical assumptions, they can all be written as special cases of the novel class of "linear-gradient systems." In Section 3 we show how these linear-gradient systems can be integrated numerically in such a way that $V(x)$ is constant or non-increasing, as appropriate.

An extended version of this work, including proofs of the results presented here, is given in [14].

\section{II. "LINEAR-GRADIENT SYSTEMS"}

Our main result is the following:

Theorem 1. Let the $O D E$

$$
\dot{x}=f(x), \quad f \in C^{r},
$$

possess a $C^{r+1}$ Morse function $V(x)$, where

(a) $\frac{d V}{d t}=0$, i.e., $V$ is an integral; or

(b) $\frac{d V}{d t} \leq 0$, i.e., $V$ is a (weak) Lyapunov function; or

(c) $\frac{d V}{d t}<0$ where $f(x) \neq 0$, i.e., $V$ is a strong Lyapunov function. 
Then for all $\{x \mid \nabla V(x) \neq 0\}$ there exists a locally bounded $C^{r}$ matrix $L(x)$ such that the ODE (6) can be rewritten in the linear-gradient form

$$
\dot{x}=L(x) \nabla V(x)
$$

where

(a) $\quad L(x)$ is an antisymmetric matrix; resp.

(b) $\quad L(x)$ is a negative semidefinite matrix; resp.

(c) $\quad L(x)$ is a negative definite matrix.

\section{Remarks:}

1. A Morse function is a function whose critical points are all nondegenerate. A negative semidefinite matrix $L$ is a matrix such that $v^{t} L v \leq 0$ for all vectors $v$. A negative definite matrix $L$ is a matrix such that $v^{t} L v<0$ for all non-zero vectors $v$.

2. Under a coordinate transformation $x \mapsto C(x)$ we have $L(x) \mapsto \tilde{L}(x):=$ $d C(x) L(x)(d C(x))^{t}$. This implies that the theorem is invariant under coordinate trans-

formations, because $\tilde{L}$ is antisymmetric, negative semidefinite, resp. negative definite iff $L$ is.

3. The theorem has a converse: if an ODE is in linear-gradient form (7) with $L$ antisymmetric, resp. negative semidefinite, resp. negative definite, then $V$ is an integral, resp. weak Lyapunov function, resp. strong Lyapunov function.

4. If the sign of $d V / d t$ (zero, nonpositive, or negative) depends on $x$, then $L$ can be chosen to be antisymmetric, negative semidefinite, or negative definite respectively, depending on $x$. The type of representation is not unique: at points where $d V / d t=0$, $L$ can be chosen to be either antisymmetric or negative semidefinite. 
5. A particular $L(x)$ satisfying the requirements of the theorem is

$$
L_{i j}(x)=\frac{f_{i} v_{j}-v_{i} f_{j}+\delta_{i j} \sum f_{k} v_{k}}{\sum v_{k}^{2}}
$$

where $v_{j}=\partial V / \partial x_{j}$. However, $L$ in (1]) yielding (6) is not unique. In particular, under further mild technical conditions, there is an $L$ which extends smoothly through critical points of $V$.

6. The fact that all systems with an integral can be written in the skew-gradient form $\dot{x}=L(x) \nabla V(x)$ was, as far as we know, first published in [16]. The general case is new, although the special case of the converse with $L(x)$ symmetric negative definite is well known and forms the subject of "generalized gradient systems" in dynamical systems [9].

The constructive proof of Theorem 1 is given in [14]. We now give some illustrative examples of the above theorem.

Example 6. Particle in 1D with friction [9]. Consider the ODE

$$
\dot{x}_{1}=x_{2}, \quad \dot{x}_{2}=-\frac{\partial f\left(x_{1}\right)}{\partial x_{1}}-\alpha x_{2},
$$

where $\alpha \geq 0$ is a coefficient of friction, and $f$ is a potential function. Eq. (9) has the energy $V\left(x_{1}, x_{2}\right)=\frac{1}{2} x_{2}^{2}+f\left(x_{1}\right)$ as a Lyapunov function, and can be written in the linear-gradient form (17) as

$$
\left(\begin{array}{c}
\dot{x}_{1} \\
\dot{x}_{2}
\end{array}\right)=\left(\begin{array}{cc}
0 & 1 \\
-1 & -\alpha
\end{array}\right) \nabla V\left(x_{1}, x_{2}\right) .
$$

For $\alpha=0$, the system is conservative, and the matrix $L$ is antisymmetric (case (a) above). For $\alpha>0$, the system is dissipative, $V$ is a (weak) Lyapunov function, and $L(x)$ is negative semidefinite (case (b) above; cf $\S 9.4$ of [7]).

Example \%. An averaged system in wind-induced oscillation [6]. Consider the system

$$
\begin{aligned}
& \dot{x}_{1}=-\zeta x_{1}-\lambda x_{2}+x_{1} x_{2} \\
& \dot{x}_{2}=\lambda x_{1}-\zeta x_{2}+\frac{1}{2}\left(x_{1}^{2}-x_{2}^{2}\right) .
\end{aligned}
$$


Here $\zeta \geq 0$ is a damping factor and $\lambda$ is a detuning parameter. Guckenheimer and Holmes [6] remark that for $\zeta=0$, Eq. (11) has the Hamiltonian

$$
V\left(x_{1}, x_{2}\right)=-\frac{\lambda}{2}\left(x_{1}^{2}+x_{2}^{2}\right)+\frac{1}{2}\left(x_{1} x_{2}^{2}-\frac{x_{1}^{3}}{3}\right),
$$

and for $\lambda=0$, Eq. (11) is a gradient system with

$$
V\left(x_{1}, x_{2}\right)=\frac{\zeta}{2}\left(x_{1}^{2}+x_{2}^{2}\right)+\frac{1}{2}\left(\frac{x_{2}^{3}}{3}-x_{1}^{2} x_{2}\right) .
$$

We now show that, for all allowed values of $\zeta$ and $\lambda$, Eq. (11) can be written in lineargradient form. To this end denote $\zeta=\rho \cos (\theta)$ and $\lambda=\rho \sin (\theta)$. Then Eq. (11) can be written in linear-gradient form $\dot{x}=L \nabla V$ with

$$
L=\left(\begin{array}{cc}
-\cos (\theta) & -\sin (\theta) \\
\sin (\theta) & -\cos (\theta)
\end{array}\right),
$$

and

$$
\begin{aligned}
V\left(x_{1}, x_{2}\right)= & \frac{1}{2} \rho\left(x_{1}^{2}+x_{2}^{2}\right)-\frac{1}{2} \sin (\theta)\left(x_{1} x_{2}^{2}-\frac{x_{1}^{3}}{3}\right) \\
& +\frac{1}{2} \cos (\theta)\left(\frac{x_{2}^{3}}{3}-x_{1}^{2} x_{2}\right) .
\end{aligned}
$$

Note that for the matrix $L$ in this example we have $v^{t} L v=-\cos (\theta)\left|v^{2}\right|$. Therefore, in the physical regime (where $\zeta \geq 0$ and hence $\cos (\theta) \geq 0$ ) either the matrix $L$ is antisymmetric and $V$ is an integral (for $\cos (\theta)=0$ ), or $L$ is negative definite and $V$ is a strong Lyapunov function (for $\cos (\theta)>0$ ). (Note that for $\lambda=\zeta=0$, we have $\rho=0$ and we are free to choose $\theta$. In this limit, the system possesses an integral

$$
V_{1}\left(x_{1}, x_{2}\right)=\frac{1}{2}\left(x_{1} x_{2}^{2}-\frac{x_{1}^{3}}{3}\right)
$$

as well as a Lyapunov function

$$
V_{2}\left(x_{1}, x_{2}\right)=\frac{1}{2}\left(\frac{x_{2}^{3}}{3}-x_{1}^{2} x_{2}\right)
$$

and $V$ given by Eq. (15) represents an arbitrary linear combination of these two functions). 
Example 8. Reproduced from [16], here is the linear-gradient form for the ODE (2) in Example 3:

$$
\left(\begin{array}{c}
\dot{x}_{1} \\
\dot{x}_{2} \\
\dot{x}_{3}
\end{array}\right)=\left(\begin{array}{ccc}
0 & 0 & -e^{x_{3}} \\
0 & 0 & -e^{x_{1}}-e^{x_{3}} \\
e^{x_{3}} & e^{x_{1}}+e^{x_{3}} & 0
\end{array}\right) \nabla V
$$

where $V$ is given by Eq. (3).

Example 9. Here is the linear-gradient form for the ODE (4) in Example 5:

$$
\left(\begin{array}{c}
\dot{x}_{1} \\
\dot{x}_{2}
\end{array}\right)=\left(\begin{array}{cc}
a & b \\
-b & a
\end{array}\right) \nabla V\left(x_{1}, x_{2}\right),
$$

where $a=-\left(x_{1}^{4}+x_{2}^{4}\right) /\left(x_{1}^{2}+x_{2}^{2}\right), b=-\left(x_{1}^{2}+x_{2}^{2}+x_{2} x_{1}^{3}-x_{1} x_{2}^{3}\right) /\left(x_{1}^{2}+x_{2}^{2}\right)$, and $V\left(x_{1}, x_{2}\right)$ is given by Eq. (5). Note that the matrix $L$ in (19) is negative definite.

\section{DISCRETE GRADIENTS AND THE NUMERICAL INTEGRATION OF LINEAR-GRADIENT SYSTEMS}

For differential equations whose time evolution has particular structural properties, such as preservation of symplectic structure, phase space volume, symmetries, or conserved quantities, it is desirable to mimic these properties in any numerical integration [20. This is

particularly useful in long-time integrations. One can also view the discrete-time analogues as interesting physical systems in their own right [12].

A major application of the linear-gradient formulation (7) is that it has a simple and elegant discrete-time analogue; moreover, this analogue is also a universal representation for systems of each class.

Definition 1. [3] Let $V(x)$ be a differentiable function. Then $\bar{\nabla} V\left(x, x^{\prime}\right)$ is a discrete gradient of $V$ if it is continuous and

$$
\begin{aligned}
& \bar{\nabla} V\left(x, x^{\prime}\right) \cdot\left(x^{\prime}-x\right)=V\left(x^{\prime}\right)-V(x) \\
& \bar{\nabla} V(x, x)=\nabla V(x)
\end{aligned}
$$


Discrete gradients are not unique. Several examples of discrete gradients are given in [3,8, 14.

Definition 2. The function $V$ is an integral of the map $x \mapsto x^{\prime}$ if $V\left(x^{\prime}\right)=V(x), \forall x$. It is a weak Lyapunov function if $V\left(x^{\prime}\right) \leq V(x), \forall x$. It is a strong Lyapunov function if $V\left(x^{\prime}\right)<V(x)$ for all $x$ such that $x \neq x^{\prime}$.

Theorem 2. [14] Let the map $x \mapsto x^{\prime}$ be defined implicitly by

$$
\left(\frac{\Delta x}{\Delta t}=\right) \frac{x^{\prime}-x}{\tau}=\tilde{L}\left(x, x^{\prime}, \tau\right) \bar{\nabla} V\left(x, x^{\prime}\right)
$$

where $\bar{\nabla} V$ is any discrete gradient, $\tilde{L}$ is a matrix function, and $\tau$ represents a timestep. Then $V(x)$ is an integral, resp. weak Lyapunov function, resp. strong Lyapunov function of the

map if $\tilde{L}$ is antisymmetric, resp. negative semidefinite, resp. negative definite. Conversely, for any map with such $a V$, and any discrete gradient $\bar{\nabla}$, at points such that $\bar{\nabla} V\left(x, x^{\prime}\right) \neq 0$ there exists an $\tilde{L}$ such that that map takes the form (21).

It follows that (21) is a discrete approximation to the linear-gradient system (7) that preserves integrals, resp. Lyapunov functions, provided the method is consistent, i.e., $\tilde{L}(x, x, 0)=L(x)$.

Equations similar to (20), (21) have appeared in many energy-conserving schemes for Hamiltonian systems [2, [1, 10,11], although the first axiomatic presentation was [3], and the first application to all systems with an integral was [17].

\section{CONCLUDING REMARKS}

(i) In this Letter, for simplicity we have restricted our discussion to the case of one first integral or Lyapunov function. In [14 we show that an $n$-dimensional ODE with $m \leq n-1$ integrals and/or Lyapunov functions $V_{1}, \ldots, V_{m}$, can be written in the "multilinear-gradient" form

$$
\dot{x}=L(x) \nabla V_{1} \ldots \nabla V_{m}, \quad x \in \mathbb{R}^{n}
$$


where $L(x)$ is an $(m+1)$-tensor. Structure-preserving integrators for Eq. (22) have also been constructed, generalizing Eq. (21).

(ii) Associated with (multi)linear-gradient systems of the form (22) there is also a formulation in terms of a bracket:

$$
\frac{d f(x)}{d t}=\left\{f, V_{1}, \ldots, V_{m}\right\}_{L}
$$

where the bracket is defined by

$$
\left\{f_{1}, \ldots, f_{p}\right\}_{L}:=\sum_{i_{1}, \ldots, i_{p}} L_{i_{1}, \ldots, i_{p}} \frac{\partial f_{1}}{\partial x_{i_{1}}} \ldots \frac{\partial f_{p}}{\partial x_{i_{p}}}
$$

where $p=m+1$. This bracket satisfies the Leibnitz rule in each of its variables:

$$
\begin{aligned}
& \left\{f_{1}, \ldots, f_{j-1}, \phi\left(g_{1}, \ldots, g_{k}\right), f_{j+1}, \ldots, f_{p}\right\}_{L} \\
= & \sum_{i=1}^{k}\left(\frac{\partial \phi}{\partial g_{i}}\right)\left\{f_{1}, \ldots, f_{j-1}, g_{i}, f_{j+1}, \ldots, f_{p}\right\}_{L},
\end{aligned}
$$

$j=1, \ldots, p$. Conversely, the tensor $L$ is defined by the fundamental brackets $L_{i_{1}, \ldots, i_{p}}=$ $\left\{x_{i_{1}}, \ldots, x_{i_{p}}\right\}_{L}$. It follows that $V(x)$ is an integral, resp. weak Lyapunov function, resp. strong Lyapunov function of the (multi)linear-gradient system (22) iff $W=0 \forall x$, resp. $W \leq 0 \forall x$, resp. $W<0$ for all $x$ such that $|\nabla V(x)| \neq 0$, where $W:=\left\{V, V_{1}, \ldots, V_{m}\right\}_{L}$. It also follows that $V$ is an integral (resp. Lyapunov function) of the system (22) iff $V_{j}$ is an integral (resp. Lyapunov function) of the system

$$
\dot{x}=\tilde{L}(x) \nabla V_{1} \ldots \nabla V_{j-1} \nabla V \nabla V_{j+1} \ldots \nabla V_{m}
$$

where

$$
\tilde{L}_{i_{1, \ldots, i_{j-1}, i_{j}, i_{j+1}, \ldots i_{m}}}=L_{i_{j}, \ldots, i_{j-1}, i_{1}, i_{j+1}, \ldots, i_{m}}
$$

Special cases of the bracket (24) are the Poisson bracket and the Nambu bracket 21].

(iii) We hope to address the numerical order of accuracy of the integrator (21) in a forthcoming publication. 


\section{Acknowledgements}

We are indebted to the Marsden Fund of the Royal Society of New Zealand, and Lottery

Science New Zealand, for their financial support. G.R.W.Q. is also grateful for a La Trobe University Large Central Grant and an Australian Research Council Small Grant supporting this project. 


\section{REFERENCES}

[1] V.I. Arnold, Mathematical Methods of Classical Mechanics, 2nd ed., Springer-Verlag, New York, 1989.

[2] A.J. Chorin, T.J.R. Hughes, J.E. Marsden, and M. McCracken, Comm. Pure Appl. Math. 31 (1978), 205-256.

[3] Oscar Gonzalez, J. Nonlinear Sci. 6(5), 449-467 (1996).

[4] L. Gotusso, Appl. Math. Comput. 17 (1985), 129-136.

[5] B. Grammaticos, J. Moulin-Ollagnier, A. Ramani, J.-M. Strelcyn, and S. Wojciechowski, Physica A 163 (1990) 683.

[6] J. Guckenheimer and P. Holmes, Nonlinear Oscillations, Dynamical Systems, and Bifurcations of Vector Fields, Springer-Verlag, New York, 1983.

[7] J.K. Hale and H. Koçak, Dynamics and Bifurcations, Springer, Berlin, 1991.

[8] Amiram Harten, Peter D. Lax, and Bram van Leer, SIAM Review 25(1) (1983), 35-61.

[9] Morris Hirsch and Stephen Smale, Differential equations, dynamical systems, and linear algebra, Academic Press, New York, 1974.

[10] T. Itoh and K. Abe, J. Comput. Phys. 77 (1988) 85.

[11] Yu.A. Kriksin, Zh. Vychisl. Mat. Fiz. 33 (1993), 206-218.

[12] T.D. Lee, J. Stat. Phys. 46 (1987) 843-860.

[13] A.M. Lyapunov, The general problem of the stability of motion, Taylor and Francis, London, 1992.

[14] R.I. McLachlan, G.R.W. Quispel, and N. Robidoux, Phil. Trans. Roy. Soc. A, submitted. 
[15] Olver, P. J., Applications of Lie groups to Differential Equations, 2nd ed., SpringerVerlag, New York, 1993.

[16] G.R.W. Quispel and H.W. Capel, Phys. Lett. A 218 (1996), 223-228.

[17] G.R.W. Quispel and G.S. Turner, J. Phys. A 29 (1996), L341-L349.

[18] N. Rouche, P. Habets, and M. LaLoy, Stability theory by Liapunov's direct method, Springer Verlag, New York, 1977.

[19] D.A. Sanchez, Ordinary Differential Equations and Stability Theory, WH Freeman, San Francisco, 1968.

[20] A.M. Stuart and A.R. Humphries, Dynamical systems and numerical analysis, CUP, 1996.

[21] L. Takhtajan, Comm. Math. Phys. 160 (1994), 295-315. 